\title{
Rapid identification of spontaneously resolving capnothorax using bedside M-mode ultrasonography during laparoscopic surgery: the "lung point" sign -two cases report-
}

\author{
Dong-Min Jang, Hyung-Seok Seo, Ji Hyun Park, Byungdoo Lee, Jun-Gol Song, and Gyu-Sam Hwang \\ Department of Anesthesiology and Pain Medicine, Asan Medical Center, University of Ulsan College of Medicine, Seoul, Korea
}

Pneumothorax during general anesthesia is more difficult to diagnose compared with that of non-anesthetized patient. Furthermore, the early diagnosis of pneumothorax is to some extent difficult due to $\mathrm{CO}_{2}$-pneumoperitoneum during laparoscopic surgery. The use of ultrasonography to diagnose pneumothorax has increased in a variety of situations, demonstrating a better diagnostic rate than conventional chest radiography. Here, we report two cases of intraoperative capnothorax that were confirmed using the M-mode "lung point" sign. However, the insertion of a chest tube could have been avoided because the spontaneous resolution of capnothorax was quickly identified using bedside lung ultrasonography. (Korean J Anesthesiol 2013; 65: 578-582)

Key Words: Laparoscopy, Pneumothorax, Ultrasonography.

The incidence of pneumothorax in association with laparoscopic surgery has been reported to vary between $0.01-3 \%$ [1-3]. However, many difficulties present in anesthetized patients during laparoscopic surgery because of increased end-tidal carbon dioxide $\left(\mathrm{ETCO}_{2}\right)$ and maximal airway pressure $\left(\mathrm{P}_{\max }\right)$ levels. In contrast with pneumothorax caused by various kinds of lung injury, capnothorax that occurs due to $\mathrm{CO}_{2}$-pneumoperitoneum often resolves spontaneously within a short period of time $[2,3]$. Therefore, if capnothorax can be diagnosed during laparoscopic surgery, the insertion of a chest tube may not be required, which avoids another invasive procedure. Here, we report two cases in which capnothorax were diagnosed at the bedside using ultrasonography. Spontaneous resolution of this condition was observed using ultrasonography, most particularly the verification of the "lung point" sign.

\section{Case Reports}

\section{Case 1}

A 40-year-old woman, $169 \mathrm{~cm}, 57 \mathrm{~kg}$, underwent laparoscopic right adrenalectomy due to an adrenal pheochromocy-

Received: October 2, 2012. Revised: November 12, 2012. Accepted: December 3, 2012.

Corresponding author: Jun-Gol Song, M.D., Ph.D., Department of Anesthesiology and Pain Medicine, Asan Medical Center, University of Ulsan College of Medicine, 388-1, Pungnap-2dong, Songpa-gu, Seoul 138-736, Korea. Tel: 82-2-3010-3869, Fax: 82-2-470-1363, E-mail: jaljary@naver.com (c) This is an open-access article distributed under the terms of the Creative Commons Attribution Non-Commercial License (http:// creativecommons.org/licenses/by-nc/3.0/), which permits unrestricted non-commercial use, distribution, and reproduction in any medium, provided the original work is properly cited. 
toma. Preoperative evaluation of the patient was unremarkable. General anesthesia was induced using thiopental sodium, fentanyl, vecuronium and sevoflurane. A 7.5 Fr 3-lumen catheter was inserted under ultrasonographic guidance (linear probe: UST-5546, 5-10 MHz, machine: Prosound SSD-4400 ${ }^{\circledR}$, Aloka Medical, Co., Ltd., Tokyo, Japan) into the right internal jugular vein, and lung sliding was confirmed in both thoraces. Next, the patient was placed into the left lateral decubitus position. Before incision, $\mathrm{ETCO}_{2}, \mathrm{P}_{\max }$, and peripheral oxygen saturation $\left(\mathrm{SpO}_{2}\right)$ were determined to be $33 \mathrm{mmHg}, 17 \mathrm{cmH}_{2} \mathrm{O}$, and $100 \%$, respectively. Pneumoperitoneum was established using $\mathrm{CO}_{2}$, intraabdominal pressure was maintained $<15 \mathrm{mmHg}$, and $\mathrm{P}_{\max }$ was 23 $\mathrm{cmH}_{2} \mathrm{O}$ at the time. Thirty minutes after inducing pneumoperitoneum, the $\mathrm{ETCO}_{2}$ and $\mathrm{P}_{\text {max }}$ levels gradually increased to 43 $\mathrm{mmHg}$ and $30 \mathrm{cmH}_{2} \mathrm{O}$, respectively. Thereafter, $\mathrm{SpO}_{2}$ decreased acutely to $92 \%$. On auscultation, the breath sounds from the left lung field were normal. However, the right side of the chest demonstrated no sounds. Immediately, the fraction of inspired oxygen $\left(\mathrm{FiO}_{2}\right)$ increased from 0.5 to 0.8 , and the endotracheal tube was withdrawn back to $2 \mathrm{~cm}$ in order to rule out endobronchial intubation. Because the depth of the endotracheal tube was 18 $\mathrm{cm}$ at the incisor teeth and confirmed the depth of endotracheal tube by cuff palpation at the sternal notch, we supposed that it was enough to rule out one-lung intubation without using bronchoscope. $\mathrm{SpO}_{2}$ was maintained at between $98-100 \%$, though the right thorax was still silent. Pneumothorax was strongly suspected, and the chest was examined using ultrasonography. Lung sliding and seashore sign were demonstrated in the left lung (Fig. 1A), however there was no sliding with barcode sign in the right lung (Fig. 1B). Although the surgeon simultane-
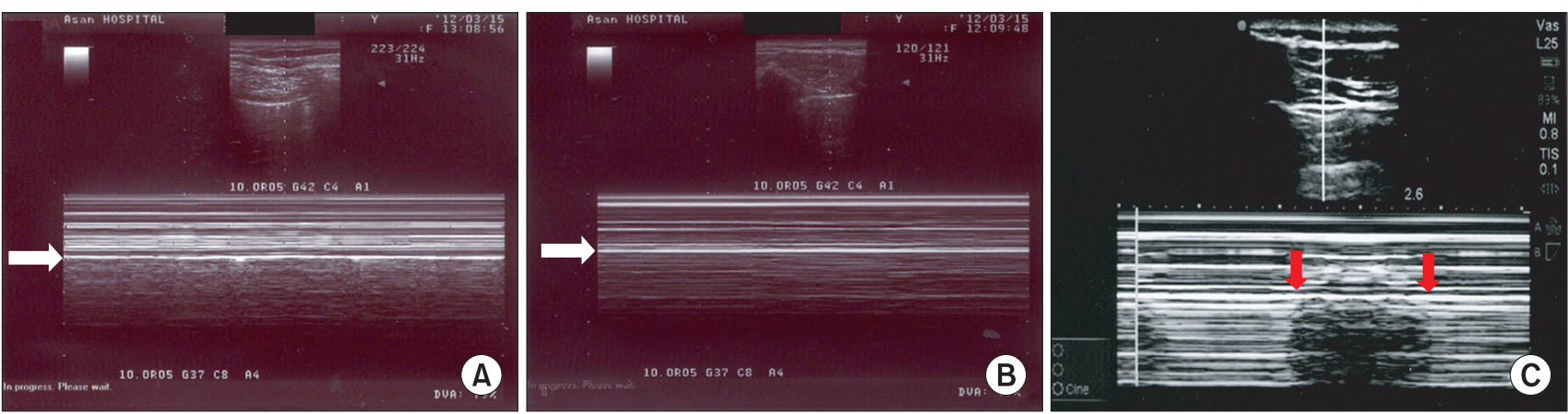

Fig. 1. M-mode images of the cases. (A) "Seashore sign". (B) "Barcode sign" (or stratosphere sign). The white arrow in each figure indicates the pleural line. Below the pleural line, the granular or sandy appearance represents normal lung sliding (A), and the straight horizontal line, which resembles a barcode (B) suggests pneumothorax. (C) The "lung point" sign in case 2. The white arrows in the figure indicate the boundary points of the seashore and barcode signs.

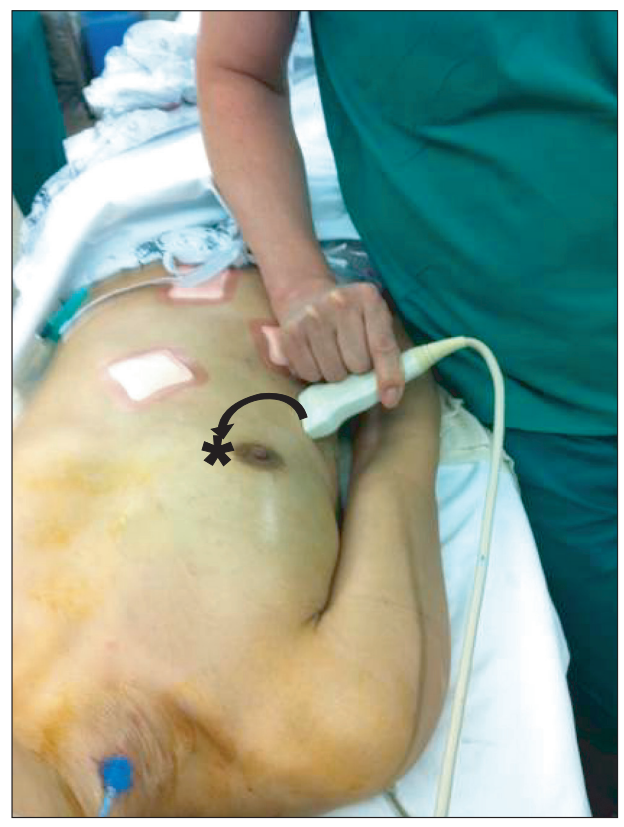

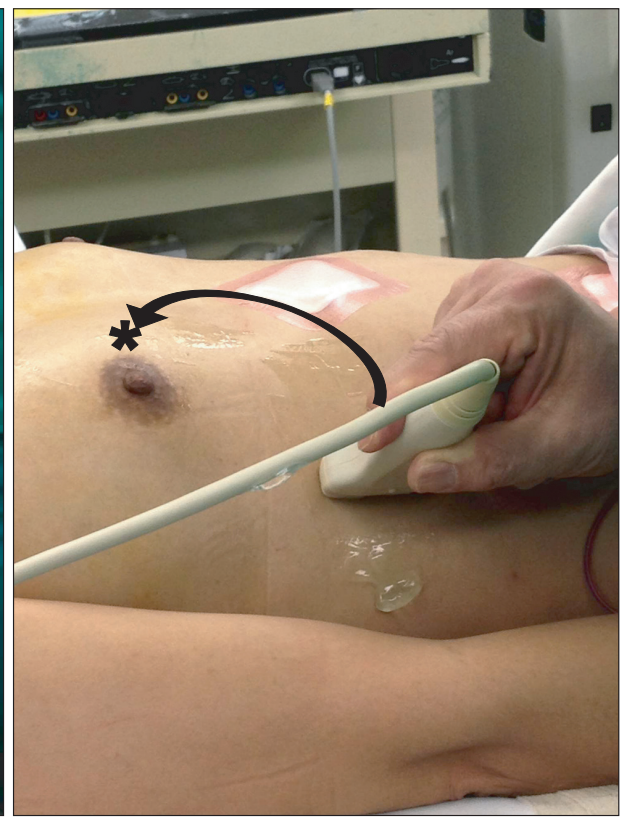

Fig. 2. Placement of an ultrasonographic probe in case 1. Movement of the "lung point" sign, which follow the warped arrow to the asterisk, means decrease of capnothorax in each figure. 
ously inspected the diaphragm in detail, no apparent injuries or defects were found. Because the vital signs were stable and $\mathrm{SpO}_{2}$ and $\mathrm{ETCO}_{2}$ were normal, as indicated by an increased $\mathrm{FiO}_{2}$ and respiratory rate, the operation was laparoscopically completed.

When the operation was concluded, a positive end-expiratory pressure (PEEP) of $5 \mathrm{cmH}_{2} \mathrm{O}$ was applied to inflate the collapsed lung. The patient was examined using ultrasonography whilst in the supine position, and at the same time, portable chest radiography was called to assess a pneumothroax. The ultrasonographic probe was placed on the right lower lateral chest wall between anterior and mid axillary line (Fig. 2), and we identified the "lung point" sign, which can be used to diagnose pneumothorax [4]. Also, chest radiography indicated a $<30 \%$ collapse of the right lung (Fig. 3A). As the right hemithorax was continuously examined using ultrasonography, auscultation of the right lung improved, as well as the appearance of lung sliding sign and the disappearance of the lung point sign in whole right hemithorax within about 30 minutes after the end of the operation. No hemodynamic instability was noted, and $\mathrm{SpO}_{2}$ was maintained at $100 \%$ using only medical air. Hence, pneumthorax was considered to be capnothorax, and the patient was extubated without the insertion of a chest tube. The patient was transferred to the postanesthetic care unit (PACU) and did not complain about any respiratory disturbance.

\section{Case 2}

A 39-year-old man, $165 \mathrm{~cm}, 63 \mathrm{~kg}$, was scheduled for living right donor nephrectomy via hand-assisted laparoscopic surgery (HALS). The patient was normal on preoperative evaluation and presented without a specific medical history. The induction of anesthesia with thiopental sodium, fentanyl, vecuronium and desflurane was uneventful. The patient was placed in the left lateral decubitus position. After $\mathrm{CO}_{2}$-pneumoperitoneum was induced, the intraabdominal pressure was maintained $<14$ $\mathrm{mmHg}$, and $\mathrm{ETCO}_{2}, \mathrm{P}_{\text {max }}$, and $\mathrm{SpO}_{2}$ were $33 \mathrm{mmHg}, 23 \mathrm{cmH}_{2} \mathrm{O}$ and $100 \%$, respectively. When the upper pole of the kidney was approached, the diaphragm was injured by the dissector, which was immediately repaired. The surgeon inquired about the status of the right lung because the right hemidiaphragm was bulging. The lung sounds in the right chest were decreased on auscultation, and $\mathrm{ETCO}_{2}$ and $\mathrm{P}_{\max }$ were increased to $42 \mathrm{mmHg}$ and 30 $\mathrm{cmH}_{2} \mathrm{O}$, respectively. However, $\mathrm{SpO}_{2}$ was maintained at $100 \%$. At that point, we evaluated the right thorax using ultrasonography (linear probe: L25x, 6-12 MHz, machine: M-turbo System ${ }^{\circledR}$, Sonosite Inc., Bothell, WA, USA) in the operative field, and the "lung point" sign was found at the right lower anterior axillary line (Fig. 1C). Considering the location of the lung point sign, a small amount of pneumothorax was expected. However, because the hemodynamic parameters were maintained in a normal range, the operation was continued via HALS and a PEEP of 5 $\mathrm{cmH}_{2} \mathrm{O}$ was applied.

At the end of the surgery, the surgeon requested a portable chest radiography and we concurrently examined the patient using ultrasonography in the supine position. Chest radiography indicated a pneumothorax $<20 \%$ (Fig. $3 \mathrm{~B}$ ), and the range of the pneumothorax which was indicated by the lung point sign was gradually diminished. Because signs of cardiopulmonary instability were not found and $\mathrm{SpO}_{2}$ was maintained without an increase in $\mathrm{FiO}_{2}$, the insertion of a chest tube was not necessary and we safely extubated the endotracheal tube. The patient was transferred to the PACU and did not complain of any respiratory discomfort.
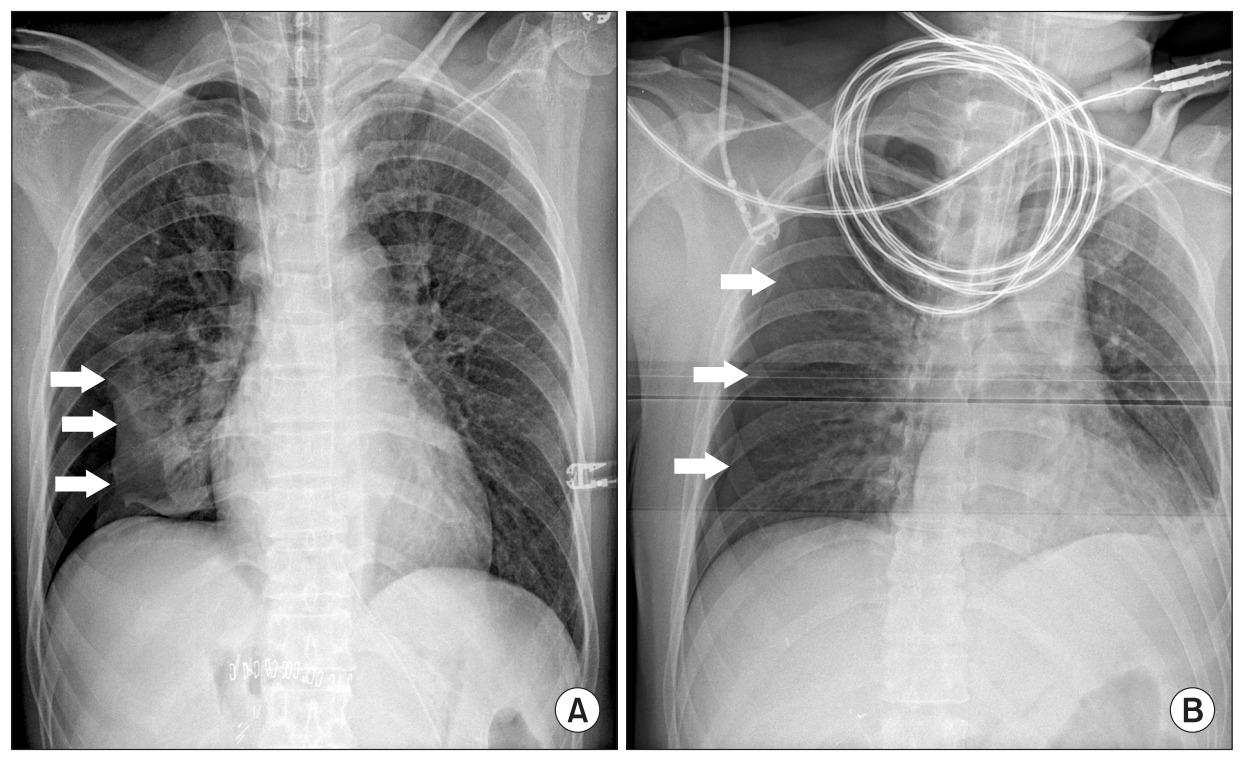

Fig. 3. Immediate postoperative portable chest radiographs in the operating room. (A) and (B) are the radiograph of case 1 and 2, respectively. Each white arrow represents the margin of pneumothorax. 


\section{Discussion}

Patients under general anesthesia may develop pneumothorax due to a variety of reasons, such as barotraumas, rupture of the lung bullae or needle insertion into the pleural cavity $[3,5]$. Capnothorax due to $\mathrm{CO}_{2}$ during laparoscopic surgery is rare, but, it has been described as a complication of almost every type of laparoscopic surgery [1-3,6-8]. The exact etiology of capnothorax still remains obscure. It has been speculated that an abnormal diaphragm caused by congenital failure of fusion (e.g., sternal, costal, and lumbar portions), weak point (e.g., around the aorta, vena cava, and esophagus), congenital defects or iatrogenic injuries by the instrument can lead to capnothorax [1-3,6]. Because the creation of pneumoperitoneum and positional changes can demonstrate several potential physiological alterations [9], the accurate diagnosis of pneumothorax is more difficult during laparoscopic surgery than other surgical procedure.

When Pneumothorax is suspected, it is traditionally diagnosed by portable chest radiography in the operating room. However, chest radiography may take a considerable amount of time and demonstrates a sensitivity of $31.8-75.5 \%$ for the diagnosis of pneumothorax [10-12]. In many different fields, lung ultrasonography is often used to diagnose pathological conditions of the lung. In particular, ultrasonography has received attention as a very effective method for confirming pneumothorax at the bedside in the operating room.

In numerous studies, ultrasonography has been reported as superior to conventional portable chest radiography for the detection of pneumothorax [4,10-13]. The presence or absence of characteristic ultrasonographic signs are key to a correct diagnosis, i.e., lung sliding, lung pulse, comet-tail artifact, barcode sign (stratosphere sign), seashore sign and lung point sign. Among these signs, lung sliding may be most widely known. It is shimmery horizontal movement of pleural line between two shadows of ribs [13]. However, the absence of lung sliding does not always indicate pneumothorax due to several mechanical factors (e.g., poor technique, inappropriate ultrasound probe frequency, the presence of dynamic noise filters) and the patient's affliction (e.g., main bronchial intubation, emphysema, atelectasis, pleural calcification, pleural adhesion, lung fibrosis) [13,14]. For these reasons, more specific ultrasonographic methods are needed to confirm pneumothorax.

In a particular area of the thorax, the ultrasonographic patterns of pneumothorax and the normal lung alternatively appear depending on the respiratory cycle. This is referred to as the "lung point" sign. This can be explained by a slight increase in pulmonary volume during inspiration and can be observed in two ultrasonographic modes: real-time (B-mode) and time-motion (M-mode) [4]. In B-mode, the lung point sign can be found by observing lung-sliding and absent of lung-sliding alternately at a same pleural line. Analogous patterns also can be found in Mmode. Seashore sign and barcode sign can be observed in turn, and these signs demonstrate the specificity of $100 \%$ for the diagnosis of pneumothorax [4]. Furthermore, by serially observing the location of the sign, the extension of pneumothorax may be identified. Despite this wide spread practice, there are few studies about the relationship between the lung point sign and the extension of pneumothorax. When it moves to the lower lateral chest wall, the volume of pneumothorax expands and the patient may need to have a chest tube immediately inserted. On the other hand, movement of the sign from the lower lateral chest to the anterior chest indicates an improvement in pneumothorax (Fig. 3). If capnothorax is highly suspected and cardiopulmonary instability is not severe, alteration of the "lung point" sign can be used to identify improvements in pneumothorax. In addition, for faster recovery of capnothorax, application of PEEP is supportive method to absorb $\mathrm{CO}_{2}$ more readily from the pleural cavity [15]. Therefore, a chest tube insertion, which can cause injury to other structures in the thorax, may not be necessary in these cases.

In conclusion, the lung point sign may predict the range and amount of pneumothorax during laparoscopic surgery. Ultrasonography can not only help to diagnose pneumothorax, but can also be used to decide treatment of pneumothorax. Most notably, in the case of capnothorax which resolves spontaneously as time goes on, the unnecessary insertion of a chest tube may be avoided by observing alterations in the lung point sign.

\section{References}

1. Prystowsky JB, Jericho BG, Epstein HM. Spontaneous bilateral pneumothorax--complication of laparoscopic cholecystectomy. Surgery 1993; 114: 988-92.

2. Hawasli A. Spontaneous resolution of massive laparoscopy-associated pneumothorax: the case of the bulging diaphragm and review of the literature. J Laparoendosc Adv Surg Tech A 2002; 12: 77-82.

3. Msezane LP, Zorn KC, Gofrit ON, Schade GR, Shalhav AL. Case report: conservative management of a large capnothorax following laparoscopic renal surgery. J Endourol 2007; 21: 1445-7.

4. Lichtenstein D, Mezière G, Biderman P, Gepner A. The "lung point": an ultrasound sign specific to pneumothorax. Intensive Care Med 2000; 26: $1434-40$. 
5. Harkin CP, Sommerhaug EW, Mayer KL. An unexpected complication during laparoscopic herniorrhaphy. Anesth Analg 1999; 89: 1576-8.

6. Azocar RJ, Rios JR, Hassan M. Spontaneous pneumothorax during laparoscopic adrenalectomy secondary to a congenital diaphragmatic defect. J Clin Anesth 2002; 14: 365-7.

7. Ludemann R, Krysztopik R, Jamieson GG, Watson DI. Pneumothorax during laparoscopy. Surg Endosc 2003; 17: 1985-9.

8. Cha SM, Jung YH, Kim DS, Kang H, Baek CW, Koo GH. Spontaneous pneumothorax during laparoscopy-assisted Billroth-I gastrectomy -A case report. Korean J Anesthesiol 2010; 58: 405-8.

9. Cunningham AJ, Brull SJ. Laparoscopic cholecystectomy: anesthetic implications. Anesth Analg 1993; 76: 1120-33.

10. Rowan KR, Kirkpatrick AW, Liu D, Forkheim KE, Mayo JR, Nicolaou S. Traumatic pneumothorax detection with thoracic US: correlation with chest radiography and CT--initial experience. Radiology 2002; 225: 210-4.

11. Blaivas M, Lyon M, Duggal S. A prospective comparison of supine chest radiography and bedside ultrasound for the diagnosis of traumatic pneumothorax. Acad Emerg Med 2005; 12: 844-9.

12. Nagarsheth K, Kurek S. Ultrasound detection of pneumothorax compared with chest X-ray and computed tomography scan. Am Surg 2011; 77: 480-4.

13. Lichtenstein DA, Menu Y. A bedside ultrasound sign ruling out pneumothorax in the critically ill. Lung sliding. Chest 1995; 108: 1345-8.

14. Ueda K, Ahmed W, Ross AF. Intraoperative pneumothorax identified with transthoracic ultrasound. Anesthesiology 2011; 115: 653-5.

15. Joris JL, Chiche JD, Lamy ML. Pneumothorax during laparoscopic fundoplication: diagnosis and treatment with positive end-expiratory pressure. Anesth Analg 1995; 81: 993-1000. 